Pacific Journal of Mathematic

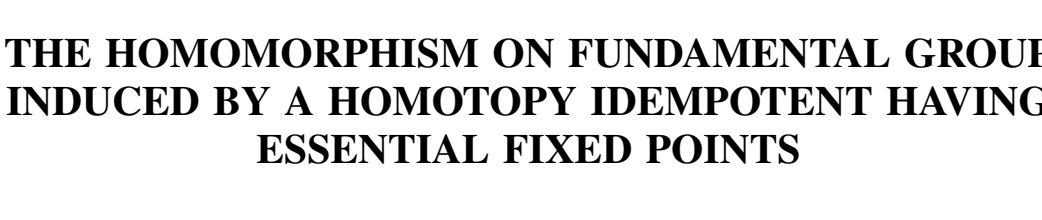




\title{
THE HOMOMORPHISM ON FUNDAMENTAL GROUP INDUCED BY A HOMUTOPY IDEMPOTENT HAVING ESSENTIAL FIXED POINTS
}

\author{
Ross GeoghegaN
}

\begin{abstract}
$f: X \rightarrow X$ is a periodic homotopy idempotent if $f$ is homotopic to $f^{k+1}$ for some positive integer $k$. Special cases are homotopy idempotents $(k=1)$ and period $k$ homeomorphisms. Let $X$ be a compact polyhedron and let $f$ have an essential fixed point $x$; there is such, for example, when the Lef schetz number is nonzero. During a homotopy $H: f \cong f^{k+1}, x$ traces out a loop $\omega$. Generalizing a theorem of Gottlieb, we show (Theorem 1.2) that the possible values of $[\omega]$ in $\pi_{1}(X, x)$ are severely restricted. In particular, some power of $f_{\sharp}([\omega])$ is a commutator. The theorem is applied in a sequel paper.
\end{abstract}

1. Introduction. In [2], Gottlieb proved that if the group $\pi$ has a finite complex as $K(\pi, 1)$ and if the Euler characteristic of $K(\pi, 1)$ is nonzero, then $\pi$ has trivial center. The core of Gottlieb's proof was the following

Proposition 1.1 (Gottlieb). Let the compact connected polyhedron $X$ have nonzero Euler characteristic, let $x \in X$, and let $\omega$ be the loop at $x$ traced out by $x$ during some homotopy which begins and ends at the identity map. Then, in $\pi(X, x),[\omega]=1$.

The main theorem in this paper is essentially a generalization of Proposition 1.1. Let us call the map $f: X \rightarrow X$ a periodic homotopy idempotent if $f$ is homotopic to $(\cong) f^{k+1}$ for some positive integer $k$. The most important case is $k=1$, when $f$ is a homotopy idempotent. Our theorem requires that an essential fixed point of $f$ be used as base point. We carefully review the relevant fixed point theory of Reidemeister, Nielsen and Wecken, in $\S 2$; for now it is enough to say that if the Lefschetz number of $f$ is nonzero, then $f$ has at least one essential fixed point. Gottlieb, in Proposition 1.1, was looking at the case $f=1_{x}$ : in that case all points are fixed points, and either all are essential or all are inessential depending on whether the Euler characteristic is nonzero or zero. Here is our theorem:

THEOREM 1.2. Let $f: X \rightarrow X$ be a periodic homotopy idempotent on a compact polyhedron, and let $x$ be an essential fixed point of $f$. Using $x$ as base point let $H: f \cong f^{k+1}$. Then there are integers $0 \leqq$ $m<n$ and a loop $\sigma$ based at $x$ such that, in $\pi_{1}(X, x),[\omega]^{n-m}=$ 
$[\sigma] \cdot f_{\sharp}^{n k+1}\left([\sigma]^{-1}\right)$. Hence when $k=1 f_{\#}\left([\omega]^{n-m}\right)$ is the commutator $f_{\#}([\sigma]) \cdot[\omega]^{-n} \cdot f_{\#}([\sigma])^{-1} \cdot[\omega]^{n}$.

Theorem 1.2 is proved in $\S 5$. A simpler version, Theorem 3.1, is proved first, because it illustrates the method while it avoids technical difficulties. In fact Gottlieb's Proposition 1.1 is a corollary of Theorem 3.1, while only the weak conclusion $[\omega]^{n-m}=1$ can be drawn from Theorem 1.2, when $f=1_{x}$. Nonetheless 1.2, and not 3.1 , is the useful result.

It has already been hinted that Theorem 1.2 is a theorem in fixed point theory, but the applications we have in mind for it are outside fixed point theory. The first application is to the problem of splitting homotopy idempotents on finite complexes. The background material for that problem is disjoint from the background material for Theorem 1.2. Hence we have separated the two: that application is made in our paper [3], which is intended to be a sequel to the present paper. However, to give a context for Theorem 1.2, we will say where homotopy idempotents actually arise. Suppose $X$

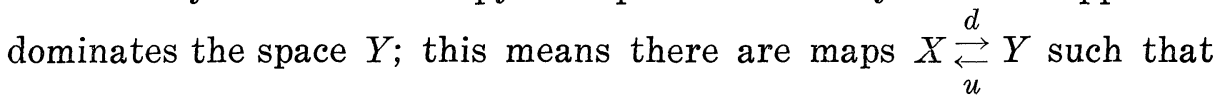
$d \circ u \cong 1_{Y}$. Then $u \circ d \cong u \circ(d \circ u) \circ d$, so $u \circ d$ is a homotopy idempotent. The problem of splitting, mentioned above, is the problem of whether this is the only source of homotopy idempotents, when $X$ is a compact polyhedron. As we write, the answer is unknown. See [3].

We have a possible second application of Theorem 1.2 in mind: it is unclear whether the Nielsen number (see $\S 2$ ) of a homotopy idempotent can exceed 1; and the answer seems to be related to the presence of $Z$-summands in $\widetilde{K}_{0}\left(\pi_{1}(X, x)\right)$. We will develop this in a later paper. (See note added in proof.)

Finally, we remark that our interest is in homotopy idempotents rather than periodic homotopy idempotents. Nonetheless, it seems sensible to do Theorem 1.2 for periodic homotopy idempotents. The only addition to the price is the need to use the classical Dirichlet theorem on the existence of infinitely many primes of the form $n k+1$, for given $k$. And periodic homeomorphisms are thereby included, though we have not yet found an application of 1.2 in that context.

We gratefully acknowledge the help of Po-chu Chiang ( $\equiv$ Bo-ju Jiang) who read an earlier version of this paper and made many helpful comments.

2. Review of fixed point theory. We recall the principal concepts of Nielsen-Reidemeister-Wecken fixed point theory. For details see [1]. 
Let $f: X \rightarrow X$ be a map on a compact connected polyhedron. Two fixed points $x$ and $y$ are f-equivalent if there is a path $\tau$ from $x=\tau(0)$ to $y=\tau(1)$ such that $\tau$ and $f \circ \tau$ are homotopic rel $\{0,1\}$. The $f$-equivalence classes are called fixed point classes: there are finitely many, and each is a closed set.

With each class is associated an integer called its index. The definition is homological, and is complicated to state in general (see [1; p. 87]). But we will only need specifics for nice maps: in that case there is a combinatorial definition which we now give. Suppose $X$ is a PL $n$-manifold in $\boldsymbol{R}^{n}$ and that for some triangulation $K$ of $X$ $f$ is affine on each simplex of $K$ and has no fixed points in the $(n-1)$-skeleton $K^{n-1}$. Let $x \in \AA$ be a fixed point in the $n$-simplex $s$

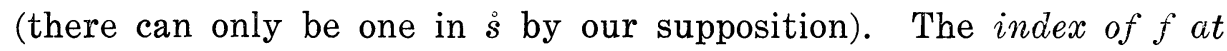
$x$ is $(-1)^{e(x)}$ where $e(x)$ is the number of real eigenvalues greater than 1 of the linear map $T_{x}$ defined by translating $f \mid \stackrel{s}{s}$ to the origin, $x$ being moved to 0 . Of course eigenvalues are to be counted with multiplicity. Equivalently, the index of $f$ at $x$ is $\operatorname{sign} \operatorname{det}\left(I-T_{x}\right)$ : the equivalence can be proved using Jordan canonical forms. The index of a fixed point class is then the sum of the indices of $f$ at each $x$ in the class.

In the general case, the index of $f$ at individual fixed points is not defined, only the index of each fiexd point class.

A fixed point class is essential if its index is nonzero. The number of essential fixed point classes is $N(f)$, the Nielsen number of $f$. We denote the set of essential fixed point classes by $E(f)$.

We now describe the sense in which $N(f)$ and the nonzero indices are homotopy invariants. Suppose $H$ is a homotopy from $f$ to $g$. Then $H$ induces an index-preserving bijection $\alpha_{H}: E(f) \rightarrow E(g)$ in the following way. First, suppose $f(x)=x$ and $g(y)=y . \quad x$ is H-related to $y$ if there is a path $\sigma$ from $x=\sigma(0)$ to $y=\sigma(1)$ such that $\sigma(\cdot)$ and $H(\sigma(\cdot), \cdot)$ are homotopic rel $\{0,1\}$. (One should think of $\sigma(t)$ as being an "up to homotopy" fixed point of $H_{t}$.) A fixed point class $F$ of $f$ is $H$-related to a fixed point class $G$ of $g$ if some (equivalently any) $x \in F$ is $H$-related to some (equivalently any) $y \in G$. It is a theorem [1; p. 94] that if $F \in E(f)$ then there is exactly one $G \in E(g)$ which is $H$-related to $F$; moreover the $f$-index of $F$ equals the $g$-index of $G$. Define $\alpha_{H}(F)=G$. Clearly we have

Proposition 2.1. If $H^{\prime}$ is a homotopy from $g$ to $h$ and if $H * H^{\prime}$ is the concatenation of $H$ and $H^{\prime}$ (from $f$ to $h$ ) then $\alpha_{H * H^{\prime}}=$ $\alpha_{H} \circ \alpha_{H}$.

The sum of the indices of all fixed point classes is the Lefschetz number $L(f)$. Of course, if $X$ is simply connected then there is only 
one fixed point class, whose index is $L(f)$.

The geometrical meaning of $N(f)$ is that for nice $X$ (e.g., manifolds of dimension $\geqq 3) f$ is homotopic to a map having $N(f)$ fixed points, and this is best possible. We shall not use this.

By an essential fixed point of $f$ we mean a fixed point whose $f$-equivalence class is essential. As we have seen, if $L(f) \neq 0, f$ has an essential fixed point. The converse is false. The map $f$ on the "figure eight" which sends $a$ to $a^{-1}$ and $b$ to $b^{2}$ ( $a$ and $b$ being the two loops) has $L(f)=0$ but both fixed points are essential [1; p. 112].

Proposition 2.2. Let $x \in X$ be a fixed point of both $f$ and $g$, let $H: f \cong g$ and $\omega=H(x, \cdot)$. If the $f$-equivalence class of $x$ is $H$-related to the g-equivalence class of $x$ then there exists a loop $\sigma$ based at $x$ such that, in $\pi_{1}(X, x)$, we have $[\omega]=[\sigma] \cdot g_{\sharp}[\sigma]^{-1}$.

Proof. For any loop $\sigma$ in $X$, the loop $(x, \cdot) *(\sigma(\cdot), 1) *(\bar{\sigma}(\cdot), \cdot)$ is homotopically trivial in $X \times I$, where $\bar{\sigma}(t) \equiv \sigma(1-t)$. Hence $\omega$ * $(g \circ \sigma) * H(\bar{\sigma}(\cdot), \cdot)$ is trivial in $X$. By hypothesis $\sigma$ can be chosen so that $H(\bar{\sigma}(\cdot), \cdot)$ is homotopic to $\bar{\sigma}$.

3. A first theorem, which implies Proposition 1.1. We now make our first application of the fixed point theory reviewed in $\S 2$ to periodic homotopy idempotents. The theorem we get, Theorem 3.1 , is not really suitable for our purposes, because the hypotheses are too strong. However: it is easily proved, it illustrates the method of proof of our "better" Theorem 1.2, and it implies Gottlieb's Proposition 1.1.

THEOREM 3.1. Let $f: X \rightarrow X$ be a periodic homotopy idempotent on a compact polyhedron and let $x$ be an essential fixed point of $f$. Using $x$ as base point, suppose there exists a pointed homotopy $F$ : $f \cong f^{k+1}$. Let $H: f \underset{\bar{\omega}}{\cong} f^{k+1}$ be another homotopy. Then there are integers $0 \leqq m<n \leqq N(f)$ and a loop $\sigma$ based at $x$ such that, in $\pi_{1}(X, x)$,

$$
[\omega]^{n-m}=[\sigma] \cdot f_{\sharp}^{n k+1}\left([\sigma]^{-1}\right) .
$$

[Compare 3.1 with 1.2. In 1.2 we do not assume that any base point preserving homotopy exists, and we cannot conclude that $n \leqq N(f)$.

Proof of 3.1. The existence of $F$ means that $x$ as a fixed point of $f^{k+1}$ is essential, since it is $F$-related to $x$ as a fixed point of $f$. Hence $x$ is an essential fixed point of $f^{r k+1}$ for all $r>0$. Now 
consider

$$
E(f) \stackrel{\alpha_{01}}{\longrightarrow} E\left(f^{k+1}\right) \stackrel{\alpha_{12}}{\longrightarrow} \cdots \stackrel{\alpha_{N-1 N}}{\longrightarrow} E\left(f^{N k+1}\right)
$$

where $N=N(f)$ and $\alpha_{i, i+1}$ abbreviates $\alpha_{H \circ\left(f^{\left.i k_{\times 1}\right)}\right.}$ (in the notation of $\S 2$ ). These are $N$ bijections between $N$-element sets each of which has a preferred element, the class of $x$. For some $m<n \alpha_{n-1, n} \circ \ldots \circ$ $\alpha_{m, m+1}$ maps the $f^{m k+1}$-class of $x$ to the $f^{n k+1}$-class of $x$. So $x$ is $\bar{H}$ related to itself, where $\bar{H}$ is the concatenation $\left(H \circ\left(f^{m k} \times 1\right)\right) * \cdots *$ $\left(H \circ\left(f^{(n-1) k} \times 1\right)\right)$. The theorem follows, by Proposition 2.2, since $\bar{H}: f^{m k+1} \underset{\omega^{\bar{n}} m}{\widetilde{\widetilde{n}}} f^{n k+1}$.

Proposition 1.1 is an immediate corollary of Theorem 3.1. Just set $f=1_{X}$; all $x$ are $1_{X}$-equivalent, so all are essential because $\chi(X) \neq 0$. If $X$ is a $K(\pi, 1)$, obstruction theory allows one to build a homotopy $H: 1_{X} \underset{\omega}{\cong} 1_{X}$ if and only if $[\omega]$ is in the center of $\pi \equiv \pi_{1}(X, x)$; hence Gottlieb's theorem that $\pi$ has trivial center. Note that Stallings [7] reproved this by a method which does not give Proposition 1.1.

4. Fixed points of iterates of a map. The purpose of this section is to prove

Proposition 4.1. Let $p$ be an odd prime and $f: X \rightarrow X$ a map on a compact polyhedron. Let $G$ be an essential fixed point class of $f^{p}$. Then at least one of following is true: (a) $N\left(f^{p}\right) \geqq p$; (b) $p$ divides the $f^{p}$-index of $G$ (c) $G$ contains an essential fixed point of $f$.

In $\S 5$ we will apply this to a periodic homotopy idempotent $f$, in which case (a) and (b) must fail for suitable large $p$, forcing (c).

To prove 4.1 we need some lemmas.

Lemma 4.2. Let $f: X \rightarrow X$ be a map on a compact PL $n$-manifold in $\boldsymbol{R}^{n}$ and let $\varepsilon>0$. There is a triangulation $K$ of $X$ and $a$ map $g: X \rightarrow X$ such that (i) $g$ is uniformly within $\varepsilon$ of $f$ and $g(X) \subset \operatorname{int} X$; (ii) $g$ is affine and nonsingular on each simplex of $K$; and (iii) the periodic points of $g$ lie in the interiors of $n$-simplexes of $K$, and those of period $p$ are isolated, for any $p$.

This is a general position lemma. In preparation for the proof we look at the affine case. If $\alpha: \boldsymbol{R}^{n} \rightarrow \boldsymbol{R}^{n}$ is affine, call the associated linear $\operatorname{map} T_{\alpha}(\cdot) \equiv \alpha(\cdot)-\alpha(0)$. Note that $T_{\beta} \circ T_{\alpha}=T_{\beta \circ \alpha}$.

Lemma 4.3. If $\operatorname{det}\left(T_{\alpha}-I\right) \neq 0$ then $\alpha$ has exactly one fixed point. 
Proof. $\alpha$ fixes $\left(I-T_{\alpha}\right)^{-1} \alpha(0)$. Suppose $\alpha$ fixes $y$ and $z ; T_{\alpha}(y-z)=$ $y-z$ so $y=z$ since $T_{\alpha}-I$ is nonsingular.

Proof of Lemma 4.2. By [4; p. 94] we can find $K$ and $g$ satisfying (i) and (ii); this is the $K$ we want, so just assume $f$ satisfies (i) and (ii). $K$ has $r$ vertices and $s n$-simplexes.

Let $\boldsymbol{F}$ be the space of maps $X \rightarrow \boldsymbol{R}^{n}$ which are affine on each $n$-simplex of $K$. Then $f \in \boldsymbol{F}$ and $\boldsymbol{F} \cong \boldsymbol{R}^{n r}$. Let $\boldsymbol{A}$ be the space of affine maps $\boldsymbol{R}^{n} \rightarrow \boldsymbol{R}^{n}$. Let $M_{n}(\boldsymbol{R})$ be the space of real $n \times n$ matrices.

We will show that for each integer $p>0$ the elements of $\boldsymbol{F}$ having isolated period $p$ points all in the interiors of $n$-simplexes are open and dense in $\boldsymbol{F}$. By the Baire Category Theorem this is enough.

We construct a nonzero polynomial map

$$
\dot{\phi}=\phi_{4} \circ \phi_{3} \circ \phi_{2} \circ \phi_{1}: \boldsymbol{R}^{n r} \cong \boldsymbol{F} \longrightarrow \boldsymbol{R}
$$

as follows. $\phi_{1}: \boldsymbol{F} \rightarrow \boldsymbol{A}^{s}$ maps $h$ to $\left(\alpha_{1}, \cdots, \alpha_{s}\right)$ where $\alpha_{i}$ is the affine map defined by $h \mid \sigma_{i} . \quad \phi_{2}: \boldsymbol{A}^{s} \rightarrow M_{n}(\boldsymbol{R})^{s}$ maps $\left(\alpha_{1}, \cdots, \alpha_{s}\right)$ to $\left(A_{1}, \cdots, A_{s}\right)$ where $A_{i}$ is the matrix of $T_{\alpha_{i}} . \quad \phi_{3}: M_{n}(\boldsymbol{R})^{s} \rightarrow M_{n}(\boldsymbol{R})^{s^{p}}$ maps $\left(A_{1}, \cdots, A_{s}\right)$ to $\left\{B_{\left(i_{1}, \cdots, i_{p}\right)}\right\}$ where $\left(i_{1}, \cdots, i_{p}\right)$ ranges over the $s^{p}$ ordered $p$-tuples picked from $\{1, \cdots, s\}$, and

$$
\begin{aligned}
B_{\left(i_{1} \cdots i_{p}\right)}= & \left(A_{i_{1}} \cdots A_{i_{p}}-I\right) \\
& \times\left(A_{i_{1}} \cdots A_{i_{p-1}}+A_{i_{1}} \cdots A_{i_{p-2}}+\cdots+A_{i_{1}}+I\right) .
\end{aligned}
$$

$\phi_{4}: M_{n}(\boldsymbol{R})^{s p} \rightarrow \boldsymbol{R}$ maps $\left(B_{\left(i_{1}, \cdots, i_{p}\right)}\right)$ to $\prod_{a 11\left(i_{1}, \cdots, i_{p}\right)} \operatorname{det} B_{\left(i_{1} \cdots, i_{p}\right)}$. $\phi_{1}$ and $\phi_{2}$ are linear, $\phi_{3}$ and $\phi_{4}$ are polynomial. $\phi$ is nonzero because $\phi(0)=$ $(-1)^{n s^{p}}$. Hence $\phi^{-1}(0)$ is nowhere dense in $\boldsymbol{F}$.

If $h \in F$, the graph of $h^{p}$ lies in the union of the graphs of the maps $\alpha_{i_{1}} \circ \cdots \circ \alpha_{i_{p}}$. If $\phi(h) \neq 0$ then each of those compositions has one fixed point, by Lemma 4.3 , so the period $p$ points of $h$ are isolated. The same matrices $A_{i_{1}} \cdots A_{i_{p}}$ occur for translates $h+\eta$, $\eta \in \boldsymbol{R}^{n}$, so $h+\eta$ also has isolated period $p$ points when $\dot{\phi}(h) \neq 0$. We claim that when $\phi(h) \neq 0$ and $\eta$ is in a dense open subset of $\boldsymbol{R}^{n}$, the (isolated) period $p$ points of $h+\eta$ miss the $(n-1)$-skeleton of $K$.

If $\alpha$ is affine and $\operatorname{det}\left(T_{\alpha}-I\right) \neq 0$ then, as in 4.3 , the unique fixed point $\left(I-T_{\alpha}\right)^{-1} \alpha(0)$ of $\alpha$ misses the polyhedron $P$ if and only if $\alpha(0)$ misses the polyhedron $\left(I-T_{\alpha}\right)(P)$. We apply this, with $P=K^{n-1}$, to each $\alpha_{i_{1}} \circ \cdots \circ \alpha_{i_{p}} \cdot \alpha(x)=T_{\alpha}(x)+\alpha(0)$, and by induction

$$
\alpha_{i_{1}} \circ \cdots \circ \alpha_{i_{p}}(0)=\sum_{j=0}^{p-1} T_{\alpha_{i_{1}} \cdots \cdots \alpha_{i_{j}}}\left(\alpha_{i_{i+1}}(0)\right) .
$$

If $\eta \in \boldsymbol{R}^{n}$, and $\beta_{i}=\alpha_{i}+\eta$, then $T_{\alpha_{i}}=T_{\beta_{i}}$

$$
\beta_{i_{1}} \circ \cdots \circ \beta_{i_{p}}(0)=\alpha_{i_{1}} \circ \cdots \circ \alpha_{i_{p}}(0)+\sum_{j=0}^{p-1} T_{\alpha_{i_{1}} \cdots \circ \alpha_{i_{j}}}(\eta) .
$$


Now $T_{\alpha_{i_{1}} \cdots \circ \alpha_{i_{j}}}=T_{\alpha_{i_{1}}} \circ \cdots \circ T_{\alpha_{i_{3}}}$, so the fact that $\phi(h) \neq 0$ ensures that $\sum_{j=0}^{p-1} T_{\alpha_{i_{1}} \cdots \circ \alpha_{i_{j}}}$ is nonsingular for every $\left(i_{1}, \cdots, i_{p}\right)$. Thus for a dense open set of $\eta$ 's, $\beta_{i_{1}} \circ \cdots \circ \beta_{i_{p}}(0)$ misses $K^{n-1}$, for all $p$-tuples $\left(i_{1}, \cdots\right.$, $\left.i_{p}\right)$. This proves the claim, and therefore the lemma.

If $p$ is prime, nonfixed period $p$ points of a map $g$ occur in orbits $\left\{x, g(x), \cdots, g^{p-1}(x)\right\}$ containing $p$ distinct points.

Lemma 4.4. Let $g$ be the map obtained in Lemma 4.2, $p$ a prime, and $\left\{x, g(x), \cdots, g^{p-1}(x)\right\}$ an orbit. Then the index of $g^{p}$ at $g^{r}(x)$ (see $\left.\S 2\right)$ is independent of $r$.

Proof. $g^{p}$ is affine and nonsingular on a small neighborhood of $x$, so the lemma boils down to showing that if $T_{0}, \cdots, T_{p-1}$ are linear maps on $\boldsymbol{R}^{n}$, sign $\operatorname{det}\left(I-T_{0} \circ \cdots \circ T_{p-1}\right)=\operatorname{sign} \operatorname{det}\left(I-T_{1} \circ \cdots \circ T_{p-1} \circ T_{0}\right)$, which is obvious (conjugate by $T_{0}$ ).

LEMMA 4.5. Let $g$ be the map obtained in Lemma 4.2, and let $G$ be an essential fixed point class of $g^{p}, p$ an odd prime. Then $G$ consists of entire fixed point classes of $g$, as well as nonfixed period $p$ points of $g$. If $N\left(g^{p}\right)<p$, the nonfixed period p points occur in $G$ in entire orbits.

Proof. The first part is immediate: if $g(x)=x \in G$ and $g(y)=y$, where $x$ is $g$-equivalent to $y$, then $x$ is $g^{p}$-equivalent to $y$, so $y \in G$. The second part is divided into cases. Case 1: $G$ contains a fixed point $y$ of $g$; let $x \in G$ be a nonfixed period $p$ point; $y$ is $g^{p}$-equivalent to $x$, so $g^{r}(y) \equiv y$ is $g^{p}$-equivalent to $g^{r}(x), 0 \leqq r \leqq p-1$, so the orbit of $x$ lies in G. Case 2: $G$ contains two or more elements of an orbit; then all the elements of that orbit are $g^{p}$-related because $p$ is prime. Case 3: $G$ has no fixed points of $g$ and contains exactly one member of the orbit $\left\{x, g(x), \cdots, g^{p-1}(x)\right\}$. Then $G$ contains at most one member of each orbit, because for all period $p$ points $y, g^{r}(x)$ is $g^{p}$ equivalent to $g^{r}(y)$ whenever $x$ is $g^{p}$-equivalent to $y(g(y) \neq y$ in Case 3). Furthermore the $g^{p}$-equivalence class of each $g^{r}(y)$ can contain no fixed points of $g$, by the analysis in Case 1, and only $g^{r}(y)$ from the orbit of $y$, by the analysis in Case 2. Thus in Case 3 we find $p$ disjoint $g^{p}$-equivalence classes $G, g(G), \cdots, g^{p-1}(G)$. They have the same $g^{p}$-index by Lemma 4.4. So we have $p$ essential fixed point classes of $g^{p}$, contradicting $N\left(g^{p}\right)<p$.

Lemma 4.6. Let $g$ be the map obtained in Lemma 4.2 and let $g(x)=x$. If $p$ is odd, $g$ and $g^{p}$ have the same index at $x$.

Proof. $g$ and $g^{p}$ are affine on a small neighborhood $U$ of $x$. Let 
$T_{x}$ be the linear map defined by translating $g \mid U$. If $\lambda_{i}$ is an eigenvalue of the linear map $T_{x}$ with multiplicity $m_{i}$, then $\lambda_{i}^{p}$ is an eigenvalue of $\left(T_{x}\right)^{p}$ with multiplicity $m_{i}[6 ; \mathrm{p} .171]$. If $\lambda_{i}$ is nonreal and $\lambda_{i}^{p}$ is real, the same is true of $\bar{\lambda}_{i}$, so the extra contribution to the index is an even power of -1 . If $\lambda_{i}$ is real $\lambda_{i}^{p}>1$ if and only if $\lambda_{i}>1$ since $p$ is odd. So the indices are the same.

Proof of Proposition 4.1. Replace $X$ by $X^{\prime}$, a regular neighborhood of $X$ in some $\boldsymbol{R}^{n}$, and $f$ by $f^{\prime} \equiv i \circ f \circ r$ where $r$ is a retraction $X^{\prime} \rightarrow X$ and $i$ is inclusion $X \rightarrow X^{\prime}$. The fixed point classes of $f^{\prime}$ are those of $f$, and their indices are the same, since the "commutativity" of indices [1; p. 53] equates the indices of $f^{\prime}=i \circ(f \circ r)$ with those of $f=(f \circ r) \circ i$. The same is true of $\left(f^{\prime}\right)^{p}$ versus $f^{p}$. So we need only prove the proposition for $f^{\prime}$. We now drop the primes.

By homotopy invariance $(\S 2)$ it is enough to prove the proposition for $g: X \rightarrow X$ obtained from $f$ by Lemma 4.2, $\varepsilon$ being small enough to ensure $g$ homotopic to $f$.

Suppose (a) and (c) fail to hold. Then $G$ contains no essential fixed point of $g$, and $N\left(g^{p}\right)<p$. By Lemmas 4.4-4.6, the contribution of the fixed points of $g$ lying in $G$ to the $g^{p}$-index of $G$ must be zero, while the contribution from nonfixed period $p$ points must be divisible by $p$. So (b) holds.

\section{Proof of Theorem 1.2.}

Proposition 5.1. Let $f \cong f^{k+1}$ be a peridic homotopy idempotent on a compact polyhedron. Then there are infinitely many primes $p$ of the form $r k+1$, such that every essential fixed point of $f$ is an essential fixed point of $f^{p}$.

Proof. By Dirichlet's theorem [5] there are infinitely many primes of the form $r k+1$. For all but a finite number of such primes $p, N\left(f^{p}\right)<p$ and $p$ does not divide any $f^{p}$-index. Hence every essential fixed point class $G$ of $f^{p}$ contains an essential fixed point of $f$, by Proposition 4.1. So $G$ must contain an entire essential fixed point class of $f$, since $f$-equivalence implies $f^{p}$-equivalence for fixed points of $f$. Since $f$ is homotopic to $f^{p}, N(f)=N\left(f^{p}\right)$, so there are no essential classes of $f$ left over.

Proof of Theorem 1.2. The proof is like that of Theorem 3.1, but we no longer know that $x$ is an essential fixed point of every $f^{r k+1}$. Instead, using Proposition 5.1, we have $N \equiv N(f)$ primes $p_{1}<p_{2}<\cdots<p_{N}$ of the form $p_{i}=r_{i} k+1$ such that $x$ is essential for $f^{p_{i}}$. As in that proof we consider $N$ bijections 


$$
E(f) \rightarrow E\left(f^{r_{1} k+1}\right) \longrightarrow \cdots \longrightarrow E\left(f^{r_{N} k+1}\right)
$$

and conclude that $[\omega]^{r_{j}-r_{i}}=[\sigma] \cdot f_{\sharp}^{r_{j} j^{k+1}}\left([\sigma]^{-1}\right)$.

Added in proof, April 1981. Theorem 1.2 is related to an algebraic theorem of Bass. See my paper Fixed points in finitely dominated compacta: the geometric meaning of a conjecture of $H$. Bass (especially Appendix II). The paper will appear in "Shape Theory and Geometric Topology," Lecture Notes in Math., SpringerVerlag.

\section{REFERENCES}

1. R. Brown, The Lefschetz Fixed Point Theorem, Scott Foresman, Glenview and London, 1971.

2. D. Gottlieb, A certain subgroup of the fundamental group, Amer. J. Math., 87 (1965), 840-856.

3. R. Geoghegan, Splitting homotopy idempotents which have essential fixed points, Pacific J. Math.

4. J. F. P. Hudson, Piecewise Linear Topology, W. A. Benjamin, New York, 1969.

5. W. J. Le Veque, Topics in Number Theory, volume 2, Addison-Wesley, Reading, 1956.

6. J. Satake, Linear Algebra, Marcel Dekker, New York, 1975.

7. J.R. Stallings, Centerless groups-an algebraic formulation of Gottlieb's theorem, Topology, 4 (1965), 129-134.

Received November 13, 1979. Supported in part by NSF Grant.

InTSitute FOR AdVANCEd Study

PRINCETON, NJ 08540

AND

State University of New York at Binghamton

BinghamTON, NY 13901 



\section{PACIFIC JOURNAL OF MATHEMATICS}

\section{EDITORS}

DONALD BABBITT (Managing Editor)

University of Galifornia

Los Angeles, California 90024

Hugo RossI

University of Utah

Salt Lake City, UT 84112

C. C. MOORE AND ANDREW OGG

University of California

Berkeley, CA 94720
J. DugundJI

Department of Mathematics University of Southern California Los Angeles, California 90007

R. FinN and J. Milgram Stanford University Stanford, California 94305

\section{ASSOCIATE EDITORS}

R. ARENS

E. F. BeCKenbaCh

B. H. NEUManN

F. WOLF

K. YosHIDA

\section{SUPPORTING INSTITUTIONS}

UNIVERSITY OF ARIZONA

UNIVERSITY OF BRITISH COLUMBIA

CALIFORNIA INSTITUTE OF TECHNOLOGY

UNIVERSITY OF CALIFORNIA

MONTANA STATE UNIVERSITY

UNIVERSITY OF NEVADA, RENO

NEW MEXICO STATE UNIVERSITY

OREGON STATE UNIVERSITY
UNIVERSITY OF OREGON

UNIVERSITY OF SOUTHERN CALIFONIA

STANFORD UNIVERSITY

UNIVERSITY OF HAWAII

UNIVERSITY OF TOKYO

UNIVERSITY OF UTAH

WASHINGTON STATE UNIVERSITY

UNIVERSITY OF WASHINGTON 


\section{Pacific Journal of Mathematics}

\section{Vol. 95, No. $1 \quad$ September, 1981}

John Allen Beachy and William David Blair, On rings with bounded

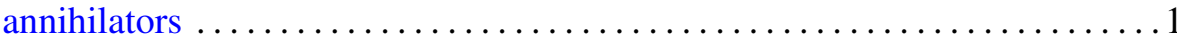

Douglas S. Bridges, A constructive look at positive linear functionals on

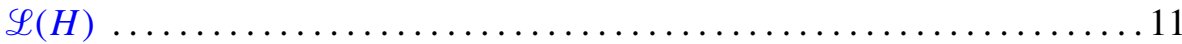

Muneo Chō and Makoto Takaguchi, Boundary points of joint numerical ranges

W. J. Cramer and William O. Ray, Solvability of nonlinear operator equations

Lester Eli Dubins and Gideon Schwarz, Equidiscontinuity of

Borsuk-Ulam functions

Maria Fragoulopoulou, Spaces of representations and enveloping 1.m.c.

*-algebras

Robert F. Geitz and J. Jerry Uhl, Jr., Vector-valued functions as families of scalar-valued functions

Ross Geoghegan, The homomorphism on fundamental group induced by a homotopy idempotent having essential fixed points

Ross Geoghegan, Splitting homotopy idempotents which have essential fixed points

Paul Jacob Koosis, Entire functions of exponential type as multipliers for

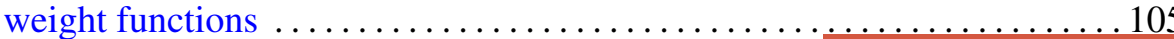

David London, Monotonicity of permanents of certain doubly stochastic matrices

Howard J. Marcum, Two results on cofibers

Giancarlo Mauceri, Zonal multipliers on the Heisenberg group

Edward Wilfred Odell, Jr. and Y. Sternfeld, A fixed point theorem in $c_{0} \quad \ldots 161$

Bernt Karsten Oksendal, Brownian motion and sets of harmonic measure zero

Andrew Douglas Pollington, The Hausdorff dimension of a set of normal numbers

Joe Repka, Base change lifting and Galois invariance ...

Gerald Suchan, Concerning the minimum of permanents on doubly stochastic circulants

Jun-ichi Tanaka, On isometries of Hardy spaces on compact abelian groups

Aaron R. Todd, Quasiregular, pseudocomplete, and Baire spaces 